\title{
Theoretical Discussion on the Treatment of Amenorrhea from the Heart and Spleen
}

\author{
Liang Liang*, Zhefeng Liu \\ Shaanxi University of Chinese Medicine, Shaanxi, China.
}

\begin{abstract}
How to cite this paper: Liang Liang, Zhefeng Liu. (2020) Theoretical Discussion on the Treatment of Amenorrhea from the Heart and Spleen. International Journal of Clinical and Experimental Medicine Research, 4(4), 260-265.

DOI: 10.26855/ijcemr.2020.10.023
\end{abstract}

Received: October 18, 2020

Accepted: November 10, 2020

Published: November 17, 2020

*Corresponding author: Liang Liang, Shaanxi University of Chinese Medicine, Shaanxi, China.

\begin{abstract}
Amenorrhea is one of the most difficult gynecological diseases in clinical practice. It seriously affects women's physical and mental health. With the increasing pollution of living environment, increasing pressure of life and the indifference of health preserving concept, incidence rate is increasing. Compared with modern medicine, the traditional Chinese medicine has obvious difference and relative superiority in understanding, diagnosing and preventing diseases. The heart and spleen are important organs of blood, which are easily affected by emotion and disorder. If the heart and spleen are not depressed, the blood flow will be smooth. Through reading a large number of ancient literature and clinical observation, this paper holds that the etiology of amenorrhea, on the whole, is nothing but the ends of deficiency and excess, obstruction of evil Qi, obstruction of cellular vessels and exhaustion of Qi and blood biochemical sources, but they are all related to the heart and spleen. The treatment of amenorrhea should be based on the heart and spleen, and the treatment methods of resolving phlegm, dispersing liver and nourishing kidney should be considered.
\end{abstract}

\section{Keywords}

Amenorrhea, Heart and spleen

In ancient Chinese medicine literature, amenorrhea is called "amenorrhea, blood is dry, the moon water is blocked, the moon water does not come, the year is not old, the menstrual water does not come", and so on. In modern medicine, amenorrhea can be divided into primary amenorrhea and secondary amenorrhea. Primary amenorrhea refers to that women have normal secondary sexual development, but no menstruation occurs when they are over 16 years old, or they have no secondary sexual development when they are over 14 years old. Secondary amenorrhea refers to the previous regular menstruation, but the menstruation is stopped for more than 6 months due to some pathological reasons, or calculated according to their original menstrual cycle More than three cycles. This discussion is mainly about secondary amenorrhea, excluding the amenorrhea caused by congenital reproductive organ development abnormalities, such as congenital absence of uterus, congenital absence of vagina, pituitary injury, hysterectomy, and physiological amenorrhea, such as pregnancy, puerperal period, etc.

Amenorrhea is a symptom of a certain disease, the etiology is more complex, and the understanding of Chinese and Western medicine is not the same. Western medicine mostly analyzes from anatomy, physiology, pathology and other aspects. The guiding ideology of the two is not the same, and the way to understand and treat the disease is also different. However, both traditional Chinese medicine and Western medicine are more difficult to treat this disease. Western medicine mostly uses hormone instead of artificial cycle therapy, which is not easy to remove roots and is easy to repeat. Compared with traditional Chinese medicine, traditional Chinese medicine has obvious 
advantages. Because of its own characteristics, traditional Chinese medicine mostly understands amenorrhea from the perspective of overall concept, mutual connection and mutual influence. The incidence rate grows with each passing day. The causes of disease can be divided into two categories: blood loss and blood stasis. With the increasing pressure of daily life, women's stress is increasing day by day, and their thinking is increasing. Since the canon of internal medicine, physicians of all ages have paid attention to the important role of the heart and spleen in the pathogenesis of amenorrhea, and in the treatment, the methods of calming the heart and strengthening the spleen, regulating qi and blood, guiding phlegm and strengthening the spleen, purging the heart and nourishing the stomach Yin, supplemented by the methods of removing blood stasis, soothing the liver, resolving phlegm and nourishing the kidney, have achieved satisfactory results in clinical practice.

\section{The oretical basis of treating amenorrhea from heart and spleen}

\subsection{Relationship between heart and spleen and Qi and blood}

\subsubsection{The heart and spleen provide sufficient Qi and blood for the five zang organs}

Holistic concept and dialectical treatment are the two basic characteristics of traditional Chinese medicine. The application of dialectical treatment is particularly prominent in gynecology of traditional Chinese medicine. To look at women's diseases, first of all, we should distinguish the differences of women's human body quality. Women use blood as their use. The "women’s complete prescription" says [1]: "to treat a disease in a large rate, first discuss its owner". Men regulate their Qi, women regulate their blood. Qi and blood, the God of human beings, should not be protected. However, women are based on blood, and their spirit is self-cleaning Women's disease, need to protect Yin blood, Qi and blood, then all diseases. Although menstruation is closely related to kidney essence, Tiangui, filling, and uterus, it ultimately depends on the sufficient Qi and blood and the coordination of the functions of the five zang organs and six Fu organs. In the five zang organs, the spleen is in charge of the transportation of water and grain. It is the source of Qi and blood biochemistry. The heart turns red, generates blood, and stores the spirit. It is the major master of the five zang organs and six Fu organs. "Spirit pivot. Determination of Qi" [2] records: "the middle energizer receives Qi to take juice, changes and becomes red, which is called blood”. When the heart is in the body and the heart functions normally, the blood vessels will be unobstructed, and the Heart Qi will promote the normal operation of blood in the vessels, playing the role of nourishing and transforming spirits. If the heart Yang is excited, the blood vessels can be operated; if the heart blood is sufficient and the mind is cultivated, the mind will be tranquil. In a word, the coordination of Qi, blood and Yin and Yang of the heart can maintain the functions of blood generation, operation and spirit storage.

When the food enters the stomach, the spleen functions normally and turns into water and grain essence. The pure body fluid Qi belongs to the heart, and the blood turns red when entering the pulse. The blood gas is sufficient and the blood is stored in the liver "The liver is the internal organ of wind and wood, because it has mutual fire, body Yin and Yang. Its strong nature and active promotion depend on the kidney water to contain it, the blood to moisten it, the lung gold to calm down, and the earth Qi of Zhonggong Dunfu to cultivate it, then the vigorous quality becomes soft body, so it can reach the smooth and luxuriant nature. What is the disease?” Deficiency of spleen and stomach leads to deficiency of biochemical source, which leads to liver failure, and heart deficiency leads to child disease and mother, and liver blood consumption. It can be seen that the normal function of the heart and spleen can provide sufficient Yin blood for the liver. Liver side can be normal catharsis, ovulation is normal. Qi and blood are active, can nourish the congenital kidney essence, kidney qi is sufficient, Tiangui is also full, secrete often, can also nourish liver yin. The lung is nourished by blood and plays a normal role in helping the heart and blood. Blood is the main substance of menstruation. The generation of menstruation depends on the sufficient of Qi and blood and the coordination of five viscera functions.

\subsubsection{Heart and spleen are the source of Chong Ren Qi and blood}

Chongmai and renmai originate from the cell and are responsible for the menstruation, pregnancy, fetus and delivery of a woman. Chong pulse is a sea of blood, a sea of five zang organs and six Fu organs. It can conserve and store Qi and blood of Zang Fu organs and connect with stomach meridian of Foot Yangming. Therefore, Chong pulse can be fed by Qi of spleen and stomach. When Taichong pulse is abundant, it can be used as the time of the month. If the heart and spleen are active and Qi and blood are sufficient, the blood and blood of the five zang organs will be abundant. If you inject Chong Ren into the meridian, you will have some profit and loss, and the month will be under the current situation. When the stomach is full of water and grain, and the function of heart generating blood is normal, then the blood of Chong pulse is abundant. “Jing Yue Quan Shu women's regulations” [3] says: "the Yin blood is the origin of Jing Yue, and there is no internal organs. Only the blood of Zang Fu organs 
belongs to Chong pulse, and Chong is the blood sea of five viscera and six Fu organs. Therefore, it is said in the Scripture that if the pulse is too strong, the moon will be in the current situation. Thus, it can be seen that Chong pulse is the origin of menstruation" [4]. The heart and spleen are the source of the twelve meridians, the sea of water and grain, the spleen and stomach are safe, and the diseases of the spleen and stomach have no biochemical source of the five zang organs and six Fu organs [5]. "Mr. Dongyuan said that the spleen is the source of Biochemistry, and the heart is the blood of all the meridians [6]. Sincerity is a word. If the heart and spleen are peaceful, the menstruation will be as usual".

\subsubsection{The heart (brain) dominates menstruation}

The brain is the fu organ of Yuan Shen and the place where yuan Shen lives. Yuan is the most important meaning. The heart governs the gods, and the brain is the residence of the gods. Though thinking is in the brain, the reason why the brain can think depends on the heart's ability to change blood to nourish it [7]. However, the mind is still in the heart, and the heart is the master of the five viscera and six viscera [8]. According to Suwen. Lingcang Mi Dian Lun: "the heart is the official of the monarch, and the gods come out". "Suwen. Tiaojing Lun" says, "the heart contains the spirit. It means that the heart plays an important role in the internal organs". "Shen" or "Shen" refers to the activity of high-level central nervous system. These functions are presided over and embodied by the heart, so it is said that "the heart controls the god" [9]. The heart (brain) is also responsible for the occurrence of menstruation. In modern medicine, there is pituitary amenorrhea with dysfunction caused by pituitary damage. Excessive mental stress, worry, fear and changes in living environment lead to dysfunction of central nervous system and hypothalamus, and amenorrhea. It can be seen that menstruation is closely related to heart (brain) [10].

\subsection{Pathogenesis basis of amenorrhea treated from heart and spleen}

\subsubsection{Heart and spleen disease can directly lead to Qi and blood without source}

The life of a lady is based on blood and Qi. If a person is ill, his blood will be hurt first. Especially for women, "Neijing” said [11]: "today's woman's life, more than gas, less than blood, with its number of blood also". No blood is needed in the course of delivery, but there are also blood injuries such as abortion, multiparty and labor injury. The profit and loss of Chong Ren blood sea is closely related to the heart and spleen. The heart qi is blocked, and the deficiency of spleen and stomach leads to the deficiency of Qi and blood. It can't nourish the liver blood, the function of liver storing blood is damaged, and the congenital kidney essence can't be fed. The kidney dominates the reproductive function, and the Qi and blood of the five zang organs are lack of source, so that the Chong and Ren are empty, and the Zang Fu organs lose the nourishment, and the function is maladjusted [12]. It can be seen that the function of liver storing blood or Chong Ren storing blood depends on the ability of heart and spleen to metabolize sufficient Qi and blood.

\subsubsection{Women are often depressed by feelings and blood}

Sun Simiao said [13]: "Women are more susceptible to diseases than men. In addition, they are deeply attached to love, love and hate, jealousy, anxiety and anger. They are strongly infected and depressed. Therefore, they are isolated from the disease and have deep roots. It is difficult to cure them”. It means that women, relative to men, are vulnerable to internal injuries of seven emotions to cause disease. They have deep obsession and are more stubborn and difficult to treat. In the seven emotions of internal injury, women often worry and worry, and most of them are depressed. If you think about hurting the spleen, you will feel qi stagnation. Qi is the commander of blood, and blood is the mother. If Qi moves, blood will flow. If Qi stops, blood will stop. So the blood knot is caused by worry and anger. And "Neijing" said [14]: "The heart is the master of the five viscera, so sad worry is the heart, heart is the five viscera shake”. All seven emotions can be used to work the mind. Restlessness makes the five organs and six organs uneasy. The heart controls blood, heart disease does not flow blood, spleen dominates taste. Food accumulation becomes visible disease, and worry becomes invisible disease. Eventually lead to heart and spleen stagnation, long and become amenorrhea.

\section{Theory and experience of modern clinical treatment of amenorrhea based on heart and spleen}

\subsection{Wang Wei et al. Treated obese polycystic ovary syndrome from heart and spleen}

Wang Wei and others believe that the occurrence and development of obese polycystic ovary syndrome is closely related to the heart and spleen, the heart qi does not fall, the spleen is not healthy movement is the key to the disease, at the same time involves phlegm dampness and blood stasis and other pathological factors, liver and stomach 
and other viscera. Clinical treatment from the point of view of the heart and spleen, mainly to the heart and spleen, combined with phlegm and blood circulation or stomach cooling blood therapy. And divided into (1) spleen deficiency and phlegm, heart stasis and blood coagulation, treatment with: Jianpi Huatan, Ning Xin Huoxue. The prescription consists of Poria cocos, Pericarpium Citri Reticulatae, Atractylodes macrocephala, Rhizoma Cyperi, radix salviae miltiorrhizae, Rhizoma Curcumae and hawthorn. The upper part can not only dissipate phlegm and invigorate spleen, but also activate blood and calm heart. (2) Phlegm dampness invading stomach, blood heat stagnation treatment to transport spleen and stomach, heart cooling blood, the main prescription Yunpi Qingxin decoction. Prescription composition: Radix Scutellariae and Rhizoma Coptidis are used to treat Salvia miltiorrhiza, radix paeoniae rubra, Rehmannia glutinosa, Uncaria rhynchophylla, forsythia, Achyranthes bidentata. The clinical effect was satisfactory [15].

\subsection{Professor Xia Guicheng's experience in treating amenorrhea}

Professor Xia Guicheng put forward the theory of "heart kidney uterus axis” through long-term clinical observation and understanding of Taiji eight trigrams. The theory holds that the heart (brain) plays a role in regulating menstruation, pregnancy, childbirth, and the days and eggs of the kidney. It also points out that the pressure of life and work of women in modern society can lead to depression of heart qi, especially the depression of liver. In the treatment of patients with amenorrhea caused by low ovarian reserve function and premature ovarian failure, the regulation and supplement of gastrointestinal function are often taken into account. Since the clinical amenorrhea patients often have poor spleen and stomach function, Qingxin Jianpi Decoction is selected to add or subtract along with the syndrome. The prescription is as follows: Xianlingpi, Xianmao each $9 \mathrm{~g}$, dangshen 15 fried Atractylodes macrocephala $10 \mathrm{~g}$, lianpi Fuling $10 \mathrm{~g}$, Gouteng $12 \mathrm{~g}$, fritillary tooth $10 \mathrm{~g}$ (first fried), cinnamon $5 \mathrm{~g}$ (later), Huanglian 5 g, guangmuxiang 6 g, Chuanduan $10 \mathrm{~g}$ [16].

\subsection{Zheng Saisai and Liu Hui used Bazhen Decoction to treat amenorrhea caused by weight loss}

Zheng Saisai and Liu Hui used Bazhen Decoction in the treatment of weight loss resulting in amenorrhea. In one case, Zheng Saisai and Liu Hui believed that amenorrhea could not be treated by three symptoms: one was that the spleen and stomach were damaged, the food was insufficient and the blood was not stopped. It was urgent to replenish the spleen and nourish blood, and the blood should be inflated to the foot meridian itself. It is considered that the amenorrhea caused by weight loss is mostly related to the deficiency of Qi and blood, and women are based on blood. The deficiency of Qi and blood can lead to the empty blood sea and the imbalance of Chong and Ren, resulting in amenorrhea. In the treatment, invigorating spleen and heart, nourishing qi and blood, regulating liver and spleen are the main treatment. The prescription includes Bazhen decoction, Sijunzi Decoction, Siwu Decoction, Radix Pseudostellariae, Radix Angelicae sinensis, Radix Paeoniae Alba, Radix Paeoniae Alba, Radix Angelicae sinensis, Radix Paeoniae Alba, Radix Angelicae sinensis, Radix Paeoniae Alba, Radix Angelicae sinensis, Radix Paeoniae Alba, Radix Paeoniae Alba, Radix Angelicae sinensis, Radix Paeoniae Alba, Radix angelicae sinensis, Radix Angelicae sinensis, Radix et Rhizoma ligustici, Radix et Rhizoma ligustici, Radix et Rhizoma ligustici, Radix Angelicae sinensis, Radix et Rhizoma, etc. Qi and blood replenishment, so that the sea of blood full and overflow, menstruation natural tide [17].

\subsection{Chuanjie and Fu Ping believed that the dysfunction of liver, heart and spleen was the important pa- thogenesis of the disease}

Chuanjie and Fu Ping believe that the deficiency of kidney essence is the key to the pathogenesis of ovarian dysfunction. Dysfunction of liver, heart and spleen is an important pathogenesis. In the aspect of medication, we pay great attention to the products of flesh and blood and affection. We think that the function of filling kidney essence of blood and meat with affection is not comparable to that of ordinary plants and trees. The commonly used drugs include Zihe Che, toad oil, donkey hide gelatin, antler glue, tortoise shell, etc. Fu Ping believes that the deficiency of heart and spleen is also an important factor causing the decline of ovarian function in patients with liver and kidney essence and blood deficiency. In the treatment, we should pay attention to nourishing the heart and spleen, and flexibly use Sijunzi Decoction and Ganmai Dazao Decoction to replenish qi and strengthen the spleen, nourish the heart and tranquilize the mind, invigorate the Qi of the stomach, nourish the fire of the heart, and blood comes from the blood [18]. 


\section{Classic case}

The patient, female, 32 years old, married, was first diagnosed on October 9, 2008. Chief complaint: amenorrhea for more than 8 months. Current medical history: one year ago, due to the family relationship was not satisfactory, depressed mood, poor appetite, insomnia and dreaminess, easy to be sleepy, easy to get angry, and not very stable mood, so he found that the menstrual volume was reduced compared with the previous period, and the amount was less than 1-2 days. The patient was 13 years old with menarche, menstrual regularity, 4/28-36 days, moderate amount and occasional dysmenorrhea. Two years ago, a natural birth of a girl, examination: admission evidence showed that the patient was thin, pale complexion, weak pulse. Light tongue, white fur, auxiliary examination: B ultrasound showed: normal pelvic cavity. Six sex hormones were normal. The body temperature was monophasic. No obvious abnormality was found in other examinations [19]. The artificial cycle was formed by using hormone, which improved and then recurred. The treatment was abandoned because of nausea and vomiting. So he went to the outpatient department of our hospital.

The last menstruation was February 2, 2018. The patient was thin. Syndrome differentiation is deficiency of Qi and blood, deficiency of both heart and spleen. Prescription: Astragalus membranaceus $10 \mathrm{~g}$, ginseng $8 \mathrm{~g}$, Atractylodes 20 g, angelica 30 g, Paeonia Alba 12 g, cypress 10 g, Polygala tenuifolia 10 g, Albizzia julibrissin 10 g, Poria cocos 12 g, Salvia miltiorrhiza 20 g, Fu Shen 10 g, Ziziphus Spinosae 10 g, Codonopsis pilosula 10 g, licorice 6 g, and told them to ease their mood. After 7 doses, the patient's appetite increased, sleep improved, spirit improved, and then clinical symptoms were added or reduced. After 4 times of treatment, the patient's face was obviously ruddy, and the spirit was good. After another clinical treatment, the patient took medicine for 4 months before and after the treatment, and her menstruation recovered, but the amount was small. She was instructed to continue taking medicine, follow up her menstruation and come to the tide on time every month.

Note. This patient is not satisfied with the family, depressed mood, thinking about the spleen, dark consumption of blood, heart and spleen deficiency, and then liver blood loss, liver Yang partial hyperactivity, liver failure, ovulation abnormalities, and Chong Ren empty, menstrual arrest. The treatment is mainly to invigorate Qi and blood, invigorate spleen and heart, supplemented by soothing liver and nourishing Yin, regulating and supplementing Chong Ren.

\section{Conclusion}

From the "Internal Classics", the disease of the two yang causes the heart and the spleen, there is no hidden song, and the female is not moon; it is passed on as the wind disappears, and the blood is dry, and it is passed on as the wind disappears. It was quoted and valued by later generations of physicians for its heart and spleen's pivotal position among the five internal organs, as well as the special physiological characteristics of women's births based on blood, and women often feel depressed, and they hurt the spleen by thinking about it. The soil in the spleen cannot be moisturized, and the soil in the spleen cannot dissipate blood. Blood is the main material basis of menstruation. The reconciliation of menstruation is sometimes related to the sufficiency of qi and blood, the balance of the functions of the five internal organs, and the fullness of urges and responsibilities. However, the heart and spleen are the source of qi and blood, provide a steady stream of acquired support for the enrichment of the kidney essence, provide a material basis for the liver to store blood, Chong Ren Sheng, and assist the lungs to assist the heart to move blood is the top priority.

The causes of amenorrhea are mostly: seven emotions, sadness, internal injury, cold, sputum stagnation, prolific abortion, chronic blood loss, etc. According to the cause and pathogenesis of amenorrhea, amenorrhea can be roughly divided into blood stagnation and blood blight, and the pathogenesis can be roughly divided into cells There are two major types of pulse obstruction and emptiness of blood vessels. Regardless of the deficiency and actuality, it is related to the heart and spleen. Cell veins belong to the heart, and both Chong and Ren arise in the cell and belong to the Yangming stomach. Not only are there inexhaustible links in the meridians, but they also influence each other in function. Therefore, most treatments start with the heart and the spleen. Xu Chunfu said [20]: The heart is yang and blood is governed, and the spleen is wrapped with blood to promote qi. If the menstruation is not smooth, it may not be due to lack of mind, thinking about hurting the spleen, fatigue, lack of energy loss, loss of nourishment of the lungs, no nourishment of kidney water, dry menstrual blood, so that the three to five are not adjusted, and gradually disappear. The testimony of wasting internal heat, bone steaming and fatigue, and the rate is difficult to treat. Only by nourishing the heart, blood is born, spleen is strong, qi is clothed, and when the two are harmonious, qi can flow smoothly. Therefore, the author believes that amenorrhea should be based on the heart and spleen, taking into account treatments such as soothing the liver, nourishing the kidney, resolving phlegm, warming 
the meridian and dredging collaterals.

\section{References}

[1] Ming.Zhang Zhongjing. (2017). Complete Book of Jingyue·Guide for Women [M]. Beijing: China Medical Science and Technology Press. 2017.12.

[2] Luan Guangyi, Yao Pengyu. (2020). Ye Tianshi's experience in treating hepatic wind based on the theory of "Yin in the liver and yang" [J]. Herald of Traditional Chinese Medicine, 2020, 26(7): 24-28.

[3] Liu Huiyan, Zhao Ruihua, Wang Fu. (2017). The Theory of “Two Yang Diseases” in Neijing and its clinical application in gynecology and andrology [J]. Beijing Traditional Chinese Medicine, 2017, 36(3): 258-260.

[4] Hong Jing, Tan Hui. (2019). Discussion on Ye Tianshi’s Academic Thoughts on Treatment of Amenorrhea [J]. Chinese Journal of Basic Medicine in Traditional Chinese Medicine, 2019, 25(02): 161-162+174.

[5] Yang Weiwei, Zhang Luoqin, Gu Ping. (2020). Observation on the curative effect of modified Erxian Decoction combined with western medicine in the treatment of premature ovarian failure and amenorrhea [J]. World Chinese Medicine, 2020, 15(06): 878-882.

[6] Zang Yunyun, Xie Qiuli, Li Saisai, et al. (2019). The treatment of amenorrhea by lifting the pot and uncovering the lid [J]. Journal of Chinese Medicine, 2019, 34(05): 931-934.

[7] Ma Shiyue, Zhu Mei. (2020). Research progress in functional hypothalamic amenorrhea [J]. Journal of International Reproductive Health/Family Planning, 2020, 39(01): 67-70.

[8] Xia Juanjuan, Tan Yingying, Huang Di, et al. (2019). A brief discussion on amenorrhea from the theory of Li Kelao's emphasis on Qi and Yuanyang [J]. Shizhen Traditional Chinese Medicine and Materia Medica, 2019, 30(07): 1745-1747.

[9] Chen Xueqi, Ge Beifen. (2018). Experience in diagnosis and treatment of secondary amenorrhea [J]. Zhejiang Journal of Traditional Chinese Medicine, 2018, 53(03): 181-182.

[10] Yang Haihao, Tang Jiqin, Han Tao, et al. (2017). Analysis of the rules of prescriptions used in the treatment of secondary amenorrhea with traditional Chinese medicine in the past 10 years [J]. Shandong Journal of Traditional Chinese Medicine, 2017, 36(02): 140-142.

[11] Wen Yinjun. (2019). Research on the academic thought and clinical experience of Ye Tianshi in treating gynecological diseases in Qing Dynasty [D]. Anhui University of Traditional Chinese Medicine, 2019.

[12] Cai Baohong, Gu Yu. (2016). On the pathogenesis and treatment of amenorrhea caused by "heart and spleen due to two Yang diseases” [J]. Journal of Liaoning University of traditional Chinese medicine, 2016, 18(03): 135-137.

[13] Wang Yang, Huang Nian, Tong Qing. (2019). Professor Chai Songyan's experience in treating amenorrhea due to weight loss [J]. Tianjin Journal of traditional Chinese medicine, 2019, 36(11): 1052-1054.

[14] Zhang Ning, Wang Tong. (2019). Analysis on the treatment of amenorrhea from "blood belongs to the heart” in Neijing [J]. Global Traditional Chinese Medicine, 2019, 12(10): 1543-1545.

[15] Chen Chunlin, Huang Xian, Yu Qingying, et al. (2019). Experience of treating ovarian amenorrhea by Luo’s gynecology in Lingnan [J]. Journal of Traditional Chinese Medicine, 2019, 60(10): 887-889.

[16] Jiang Bei, Yan Qi, Cui Hongtao, et al. (2019). Discussion on the treatment of amenorrhea in women's Department guide [J]. Chinese medicine guide, 2019, 16(06): 142-146.

[17] Shi Tongxia, Wang Xuehua. (2019). Empirical Study on the treatment of amenorrhea by syndrome differentiation of traditional Chinese medicine [J]. Contemporary Medicine Review, 2019, 17(10): 202-203.

[18] Fan Xiaoyan. (2019). Self made Huatan Tongjing Decoction in the treatment of 30 cases of amenorrhea with phlegm dampness block type [J]. Chinese Journal of Traditional Chinese Medicine Science and Technology, 2019, 26(04): 648-649.

[19] Chuanjie, Fu Ping, Zhou Feifei. (2019). Fu Ping’s experience in treating ovarian dysfunction [J]. Journal of Zhejiang University of Traditional Chinese Medicine, 2019, 43(02): 163-165.

[20] Zheng Sai, Liu Hui. (2019). An experience case of weight loss induced closure in adolescence treated with Bazhen decoction [J]. World latest medical information digest, 2019, 19(06): 267. 